\title{
DIAGNOSIS FROM COLOURS AND SMELLS
}

\author{
A Taylor, Consultant Chemical Pathologist \\ Furness General Hospital
}

Testing the urines by day and by night

Drop in reagent and see it's all right

'Negative, normal, there's nothing!' I cry

'I must have a positive, else I shall die!'

From Lament of a Chemical Pathologist, Professor D Baron

\section{INTRODUCTION}

The physical examination of a patient is a form of directed observation traditionally using inspection, palpitation, percussion and auscultation. The first, inspection, should include an assessment of a patient's colour, which even in this scientific age can tell us a great deal. We may be aware of odours but the importance of directed olfaction is not usually stressed.

There are two possible reason for this: the patient might find it offensive even if you have already explained your purpose, and the absence of an odour may be misleading as we shall see. This article will highlight the benefits of identifying colours and smells. These are properties of

\begin{tabular}{|c|c|c|}
\hline ODOUR & CONDITION & CHEMICAL \\
\hline sweet & ketosis & acetone \\
\hline fruity & alcoholism & acetaldehyde \\
\hline sweaty feet/vomitus & & $\begin{array}{l}\text { aliphatic short chain } \\
\text { fatty acids }\end{array}$ \\
\hline fishy & & $\begin{array}{l}\text { trimethylamine, } \\
\text { aliphatic amines }\end{array}$ \\
\hline halitosis & & mercaptans \\
\hline faecal & & skatole \\
\hline putrefaction & & putrescine, cadaverine \\
\hline \multicolumn{3}{|l|}{ SKIN COLOUR } \\
\hline white & pallor (albinism) & \\
\hline \multirow[t]{2}{*}{ black, grey } & solar radiation & melanin \\
\hline & ochronosis & alkapton \\
\hline \multirow[t]{2}{*}{ blue } & $\begin{array}{c}\text { peripheral/central } \\
\text { cyanosis }\end{array}$ & $\begin{array}{c}\text { chlorate poisoning } \\
\text { (haemoglobin derivatives) }\end{array}$ \\
\hline & facial cyanosis & deoxyhaemoglobin \\
\hline yellow & jaundice & bilirubin, carotenaemia \\
\hline yellow-green & & biliverdin \\
\hline red & flushing & $\begin{array}{l}\text { oxyhaemoglobin } \\
\text { erythrodontia (porphyrins) }\end{array}$ \\
\hline \multicolumn{3}{|l|}{ UROSCOPY } \\
\hline blue nappy & & indole metabolites \\
\hline \multirow[t]{2}{*}{ red to black } & haematuria & $\begin{array}{l}\text { methaemoglobin } \\
\text { haemoglobin }\end{array}$ \\
\hline & $\begin{array}{c}\text { acute intermittent } \\
\text { porphyria }\end{array}$ & pseudoporphyrin \\
\hline brown & various & $\begin{array}{c}\text { myoglobin } \\
\text { metmyoglobin/melanin/ } \\
\text { melanogens/bilirubin }\end{array}$ \\
\hline
\end{tabular}

Table 1 chemical compounds which may aid diagnosis. I shall also refer to urinalysis where colours are generated in the test tube or on a reagent stick; this is the modern equivalent of uroscopy, which was to urinalysis what astrology is to astronomy. Though occasionally light-hearted, there are important 'practice points'.

\section{ODOURS}

Odours can be immediately diagnostic: the smell of acetone in A\&E suggests that a patient has diabetic ketoacidosis. Many is the time a ward sister has surprised the new juniors with an exclamation on entering the ward - 'Ah, we have a new diabetic with us today'. People do vary in their sensitivity to the smell of acetone and many of us fail to detect it at low concentrations. We have difficulty demonstrating this sweet cloying smell to students now because most laboratories no longer stock acetone.

Medicolegal cases have been described in which the diagnosis of diabetic ketoacidosis was not made when a patient was admitted with abdominal pain. Visceral pain in this situation is actually a consequence of ketosis and early ketoacidosis is seen even with glucose levels below $16 \mathrm{mmol} / \mathrm{l}$ (euglycaemic ketoacidosis). If the glucose is low the diagnosis of diabetes could be missed. Diabetic coma can occur without ketosis (hypersmolar nonketotic diabetic coma [HONK]) and in this situation the patient's breath does not smell of acetone. The sweet smell of acetone may be detected in starvation although ketosis is rarely severe, and in isopropanol poisoning from drinking windscreen washer fluid.

Most of us can detect and identify the odour of alcohol excess, unless we have led particularly sheltered lives. This is in part the sweet fruity smell of acetaldehyde, accumulating because it cannot be catabolised quickly enough by hepatic aldehyde oxidase. Some individuals lack this enzyme and therefore are unable to tolerate ethanol. For such people, taking even a spoonful of sherry induces facial flushing and severe nausea. The Chinese have historically had a culture based on opium use rather than alcohol, probably for this reason. We know that acetaldehyde plays a role in the development of cirrhosis because it generates free radicals and certain individuals generate more than others.

Patients with alcoholic ketoacidosis characteristically are malnourished and present with abdominal pain and vomiting. Typically they have consumed a large quantity of alcohol and ketosis causes their symptoms. Dehydration and coma supervene 36-72 hours later. They may present hypothermic and stuporose at a later stage. Cases have been reported in juveniles as well as in skid-row alcoholics. The findings are metabolic acidosis, ketosis and occasionally hypoglycaemia. 
Blood alcohol levels are not usually high at the time of presentation and the patients might not smell of acetaldehyde, contributing to the failure of diagnosis. They do not smell of acetone because the ketone formed is mainly betahydroxybutric acid, the reduced ketone body which is not catabolized to acetone ${ }^{(1)}$. The stick test for urine gives a negative result and this may prompt premature exclusion of the diagnosis. Lactic acidosis and thiamine deficiency are other causes of metabolic acidosis with much worse prognosis and the outlook is worse when combined with methanol and ethanediol poisoning (antifreeze).

Case: a two-year-old child helped himself to gin. He was put to bed to sleep it off but was unrousable six hours later. His serum ethanol was only $23 \mathrm{mg} / 100 \mathrm{ml}$ on admission but the plasma glucose was less than $0.5 \mathrm{mmol} / 1$. Hypoglycaemia is the most serious complication of alcohol ingestion in small children and glucagon is contraindicated. This is because the formation of glucose from lactate in the liver is inhibited by NADH2, formed by the metabolism of ethanol. Prophylactic iv glucose is recommended for young children thus intoxicated. In this sort of case the history is important because of the absence of tell-tale odours.

Case: a 24-year-old diabetic on insulin was found dead at home. The urine alcohol was $153 \mathrm{mg} / 100 \mathrm{ml}$ and the serum glucose undetectable. Though an accidental insulin overdose cannot be ruled out, hypoglycaemia due to alcohol consumption is a possibility. These results are, however, not diagnostic because glucose may be consumed from postmortem blood in a warm body. Confirmation of a low glucose concentration in eye fluid is important in such cases and this was also undetectable. In such a case which actually presents in A\&E, an impression of excess alcohol ingestion is helpful.

Type I diabetic patients should be warned about consuming low calorie beers, spirits or dry wines since they tend to promote hypoglycaemia. Twenty percent of hypoglycaemic episodes presenting to hospital have been attributed to alcohol but this appears to be higher in younger patients, especially in this area. Though this group is theoretically glucagon-responsive, this may well be ineffective in a patient who has fasted for 24 hours. It is safer if iv glucose is administered.

Some odours appear only on the breath owing to their volatility, eg acetone acetaldehyde. In many other cases such as in inborn errors of metabolism an odour will be detectable mainly in the urine. In sick children, therefore, wet nappies are a valuable source of diagnostic information. They can be a diagnostic feature: a colleague of mine diagnosed isovaleric acidaemia whilst on a ward round at Sheffield Children's Hospital. The infant, profoundly sick and acidotic, was alive and well 11 years later on treatment. There are several such malodour syndromes which occur in infancy and childhood shown in Table $2^{(2)}$.

Case: a 53-year-old lady complained of fish odour and had a urinary trimethylamine excretion of four times the normal rate. The fact that her trimethylamine- $\mathrm{N}$-oxide was increased confirmed that this was not primary, due to absence of the metabolising enzyme. The odour of rotting fish was secondary to bacterial colonisation of the gut and was treated successfully with metronidazole ${ }^{(3)}$.

The odours of urine detected in inborn errors of metabolism are shown in Table 2. Halitosis due to bacterial formation of mercaptans may indicate severe dental and gingival disease. A metallic taste may accompany fungal infections. The malodour of vomit (isobutyric acid) can be of help in assessing an unconscious patient. Faecal odours due to skatole on the breath may indicate fistulae or incontinence. Patients are often reluctant to admit the latter because of embarrassment.

On one occasion, I remember we all gathered round to sniff the pint beer glass from which a fatal concoction had been taken by a university student. The smell of the liquid residue was that of cyanide (bitter almonds), which was later confirmed. In other poisoned patients paraldehyde methylsalicylate and heavy metals may be suspected by odour. Diabetic foot ulcers may produce putrefactive odours. This is especially useful because it indicates the presence of anaerobic bacteria; metronidazole may then be added to their medication.

\begin{tabular}{|l|l|l|}
\hline ODOUR & \multicolumn{1}{|c|}{ CONDITION } & \multicolumn{1}{c|}{ CHEMICAL } \\
\hline mousy, musty & phenylacetate & classical phenylketonuria \\
\hline curry & 2-ketovaleric acid & maple syrup urine disease \\
\hline $\begin{array}{l}\text { burnt sugar/maple } \\
\text { syrup }\end{array}$ & 2-keto isocaproic acid & MSUD \\
\hline sweaty feet & isovaleric acid & $\begin{array}{l}\text { isovaleric acidaemia } \\
\text { 3-hydroxy-3- } \\
\text { methyglutaricacidaemia } \\
\text { multiple acylCoa } \\
\text { dehydrogenase deficiency }\end{array}$ \\
\hline cat urine & 3-hydroxyisovaleric & $\begin{array}{l}\text { 3-methylcrotonylglycinuria } \\
\text { multiple carboxylase } \\
\text { deficiency }\end{array}$ \\
\hline cabbage & 2-hydroxybutyric acid & $\begin{array}{l}\text { methionine malabsorption } \\
\text { tyrosinaemia I }\end{array}$ \\
\hline rancid butter & $\begin{array}{l}\text { 2-keto-4-methiobutyric } \\
\text { acid }\end{array}$ & tyrosinaemia I \\
\hline acid & methylamalonic acid & methylmalonic aciduria \\
\hline sulphur & trimethylamine & cystinuria \\
\hline fish market & trimethylaminuria \\
\hline
\end{tabular}

Table 2 Malodour syndromes

\section{COLOURS}

We are less likely to forget diagnostic signs related to colours even if the colours are actually black and white, ie melanoid pigmentation and pallor.

Pallor as a sign needs little reiteration, being mostly related to the thickness of the skin but also a marker for fear, anaemia and, rarely, hypopituitarism and classical phaeochromocytoma paroxysms. It is notoriously absent in acute severe anaemia. In anaemia due to iron deficiency the sclerae may be bluish and in Addisonian anaemia lemon yellow due to mild jaundice.

Darkening due to melanosis occurs in Addison's disease as a consequence of high levels of ACTH, which stimulates melanocytes because it contains $\alpha \mathrm{MSH}$. Melanosis is also a feature of idiopathic haemochromatosis when it is combined with an increase in haemosiderin. Classically, in this case the colour is described as slate-grey. Confounding signs are due to racial origin and solar pigmentation, although a 12-yearold girl I once saw with Addison's disease was pigmented yet had not been out of doors for two months.

Malar flush in heart valve disease and severe myxoedema is less and less seen now. In myxoedema, thinning of the skin combined with carotenaemia generates the 'peaches and 
cream' complexion seen in longstanding cases. In hypothyroid infants, jaundice is due to hyperbilirubinaemia. Cyanotic facial fiushing is the hallmark of the carcinoid syndrome and is associated with hepatomegaly, heart valve problems and diarrhoea. Redness of the face in polycythaemia and alcohol excess are well-recognised. The brick red colour of cyanide poisoning is rare. The cherry red colour of carbon monoxide poisoning is only seen at autopsy or in moribund patients. This is because of the accompanying peripheral vasoconstriction.

A blue colour of the extremities may be due to peripheral cyanosis associated with methaemoglobinaemia seen in dapsone toxicity or with nitrosamine or chlorate poisoning (chocolate blood and dark urine) and severe acute pancreatitis.

Case: a 62-year-old man showed a blue colouration of feet and hands. He was warm in the periphery and arterial gases were normal. A methaemoglobin screen was negative. On questioning the ward staff, it transpired that his NHS blue striped pyjamas had a non-colour-fast dye which was being eluted by his sweat.

Central cyanosis gives a blue tint to mucous membranes unlike the peripheral form and is dependent upon the amount of deoxygenated haemoglobin in the arterial blood. It is best seen in daylight and an oxygen desaturation of $10-15 \%$ is detected by only half the observers. This is not sufficient for routine medical practice and so saturation monitors are essential.

Jaundice is a feature of liver disease and haemolytic anaemia. Mucous membranes show this more clearly in mild cases and a serum bilirubin of above $30 \mathrm{umol} / \mathrm{l}$ can be seen clinically. Slight jaundice can be missed but will be noticeable in the serum down to about 20umol/l. The tests used to distinguish the cause are urobilinogen in an afternoon random urine and serum haptoglobin (and reticulocyte count). Positive tests indicate a haemolytic cause. This is lemon yellow in contrast to the green yellow seen in obstruction of the biliary tract (biliverdinaemia). Liver function tests aid diagnosis (with the exception of LDH) and help to distinguish hepatitis from cholestatic and obstructive jaundice early in the disease. ALP and GGT are the prime abnormalities with values respectively over three times and six times normal with an ALT less than twice normal. In acute hepatitis, however, ALT is over ten times normal with ALP and GGT only one or two times the upper limit of normal. Conjugated bilirubin in serum is increased in cholestasis but is normal in early hepatitis and Gilbert's syndrome. The latter condition is present in $5 \%$ of the population. It is innocent and is characterised by increases on fasting to $40-50 \mathrm{umol} / 1$ at most with reduction on carbohydrate feeding to normal or less than $35 \mathrm{umol} / 1$. It may be confused with compensated haemolytic anaemia but the serum haptoglobin is reduced in such cases.

Liver disease is often present without jaundice, principally in chronic hepatitis, early cirrhosis, paracetamol poisoning, autoinumune disease and Type II hepatic failure. Being yellow may occur without jaundice. This is usually due to dietary fads with high intake of carotenes.

Case: a 6-month-old infant presented with jaundice but the serum bilirubin was $16 \mathrm{umol} / 1$ and therefore serum carotenes were measured and found to be over twice normal. The mother denied an unusual diet but lycopenes were also high, supporting the evidence for a vegetarian-tomato based diet.
This may have been a manifestation of Munchausen's syndrome by proxy.

Blue nappy syndrome occurs when tryptophan metabolites from bacteria are oxidised by mild bleach-based detergents. It is an innocent finding.

Sometimes colour changes are seen only at operation. The darkening of cartilage (ochronosis) seen in alkaptonuria is one such case. Alkapton, an abnormal pigment, is formed from homogentisic acid which cannot be oxidised due to deficiency of the oxidase. Degenerative joint disease is the main manifestation. Through a colonoscope, melanosis coli can be seen due to abuse of anthracene-based purgatives.

Red teeth are seen with hirsutism of the face and palms in Gunther's disease or congenital erythrocytic porphyria. This gave rise to the myth of werewolves (Anglo Saxon for wolfman) which drank blood. The coloration is due to deposition of porphyrins in the enamel and such patients have photosensitive rashes on the face which might bleed.

\section{UROSCOPY}

But for the discovery of albumin in the urine in Bright's disease, 'the relation of chemistry and clinical medicine might have remained unknown and valued by a few among the many but . . unrecognised and unemployed by all' (Henry Bence Jones, 1847).

Medical practice in mediaeval times centred on uroscopy and the round, half-filled glass became the symbol of the doctor. Though a very dubious practice in most hands, the addition of simple chemicals to urine now render it quite useful.

The colour of normal urine is due to uroerythrin derived from blood pigments. Brown urine may appear due to jaundice (even before the onset of clinical jaundice in the obstructive type). It may be due to myoglobin or altered blood and both these pigments show up as blood at very low concentrations on stick testing. To confirm myoglobinuria which occurs in crush syndrome and poisoning the best confirmation is by blood stick and serum CK. Here muscle necrosis and subsequent release of intracellular material may result in renal failure ${ }^{(3)}$.

Case: a 23-year-old lady had a routine ENT operation and developed abdominal pain after thiopentone anaesthesia. She vomited and became increasingly ill with hyponatraemia. After transfer to the neurology ward (with parasthesiae) a medical student noticed that her urine was dark. The senior medical staff were somewhat dismissive but the eventual diagnosis was acute intermittent porphyria.

Urine which turns brown or port wine-coloured on standing in air is characteristic of acute intermittent porphyria. This is, however, not due to a porphyrin (Greek for purple) but a polymer of porphobilinogen which forms on standing. Stabilised Haemin is now available for iv use in this condition as an emergency treatment. Urinary PBG is the diagnostic test for such patients who present with abdominal pain, psychiatric disturbance, SIADH and peripheral neuropathy ${ }^{(4)}$.

Black urine was noted as a fatal sign by John Actuarius who died in 1283. Figure 1 shows a mediaeval diagnostic guide from the colours of urine. This describes colours from 


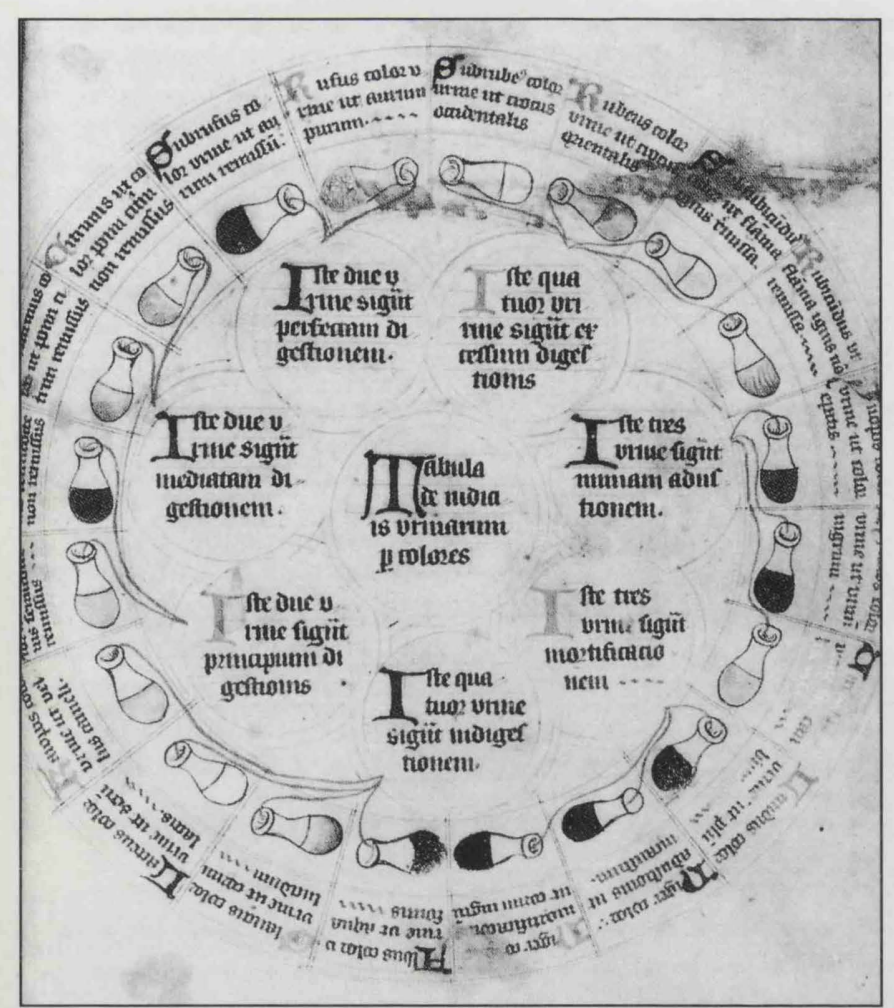

Figure 1 Mediaeval uroscopy chart, showing urine samples in various gradations of colour and condition, with suggestions to the examining doctor of what maladies are responsible

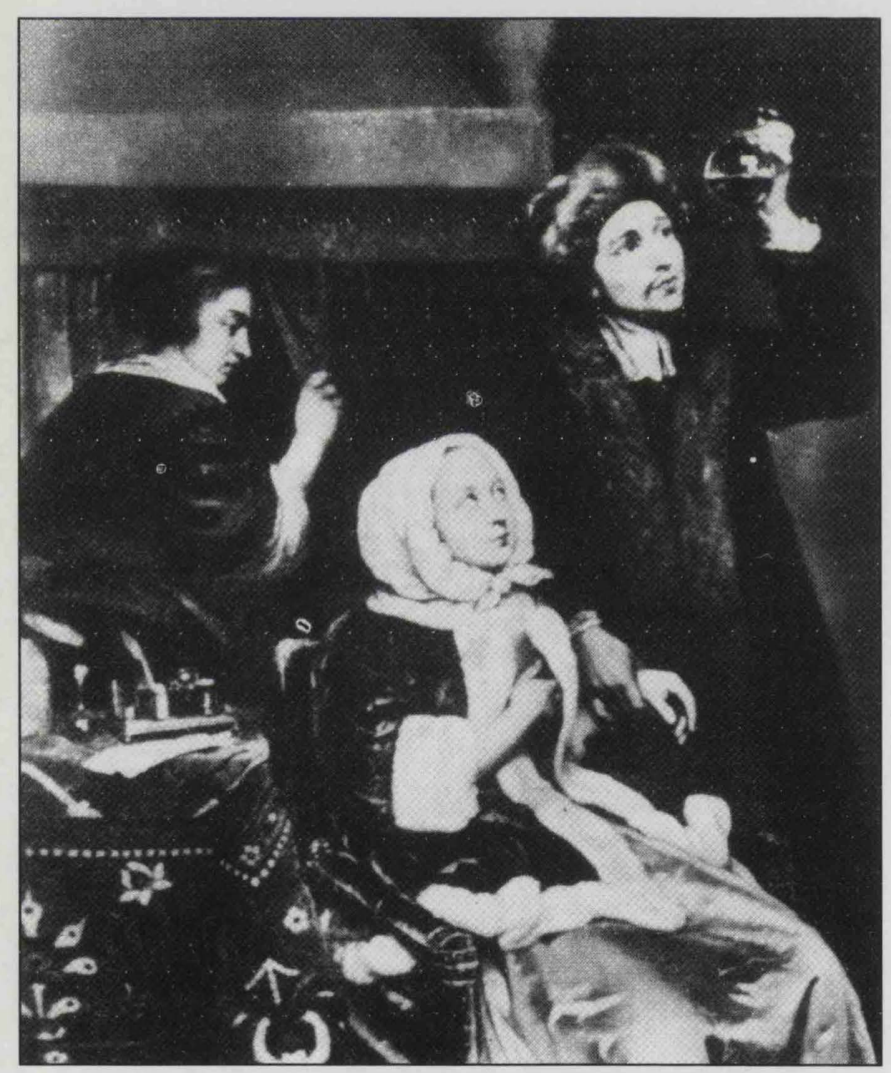

Figure 2 Illustration of a uroscopist black to red to white, and under black there is an indication of impending death. Though this may follow haematuria due to renal papillary necrosis or malaria (blackwater fever) it can be seen in malignant melanoma. In this condition ferric chloride needs to be added to convert precursors known as melanogens (principally DOPA) to melanin (a polymer). We have recently seen two cases of metastatic melanoma with positive tests for melanogens. Serum haemopexin has been used as a tumour marker where treatment needs monitoring.

Case: a 35-year-old lady presented to her GP with abnormal LFT twelve years after an ocular enucleation for a melanoma. A urine test for melanogens was positive. Extensive hepatic deposits of melanoma were found.

Urine may not look abnormal until the addition of a reagent. There are many such tests used which are the basis of modern stick tests. Some incorporate enzymes, such as tests for glucose. Ferric chloride is of particular value in metabolic screening in children and infants. The colours are of some diagnostic value as a preliminary to further definitive investigation: eg blue green in phenylketonuria, transient blue green in alkaptonuria, green grey in MSUD, green in tyrosinaemia Type I, cherry red with ketones and purple with salicylates (Table 3).

\begin{tabular}{|l|l|l|l|}
\hline REAGENT & COLOUR & URINARY ANALYTE & DISEASE \\
\hline 2-nitro-naphthol & pink & $\begin{array}{l}\text { 5-hydroxiyindole } \\
\text { acetic acide }\end{array}$ & $\begin{array}{l}\text { hepatic metastases } \\
\text { from carcinoid } \\
\text { tumour }\end{array}$ \\
\hline Ehrlich's reagent & pink & $\begin{array}{l}\text { urobilinogen/ } \\
\text { porphobilinogen }\end{array}$ & $\begin{array}{l}\text { haemolytic jaundice/ } \\
\text { porphyria }\end{array}$ \\
\hline ferric chloride & brown/black & melanogen & malignant melanoma \\
\hline cyanide nitroprusside & pink/purple & cystine & cystinuria \\
\hline
\end{tabular}

Table 3 Some examples of chromogens in urine revealed by adding a reagent

\section{CONCLUSION}

The first activity in the physical examination of a patient is inspection. Detection of colours may be of help and can be diagnostic. The presence or absence of odour may also be significant. Though we describe something overlooked as being 'under one's nose' it is well not to forget the nose itself, as an impressive analytical device.

\section{REFERENCES}

1 Wright J, Marks V Alcohol In The metabolic basis of acquired disease. Cohen et al Vol 1 p602

2 Blau N et al A physician's guide to the diagnosis of metabolic diseases. Chapman \& Hall 1996

3 Crook $\mathrm{M}$ et al Fishing for a diagnosis CPD Bulletin 2000;2(1):27

4 Burtis and Ashwood (eds) Tietz's Clinical Chemistry, 2nd edition. Saunders 1997. p2044 haematologic, p2075 porphyrins 